\title{
Factores relacionados con la permanencia estudiantil en programas de pregrado de una universidad pública'
}

\author{
Doris Amparo Parada Rico² \\ Leydi Yaneth Correa Suárez³ \\ Yeiza Fernanda Cárdenas González
}

doi:10.11144/Javeriana.ie19-1.frpe

Cómo citar: Parada Rico DA, Correa Suarez LY, Cárdenas Gonzales YF. Factores relacionados con la permanencia estudiantil en programas de pregrado de una universidad pública. Investig Enferm. Imagen Desarr. 2017;19(1): 155-170. http://dx.doi.org/10.11144/ Javeriana.ie19-1.frpe

1. Artículo original resultado de investigación. Fecha de recepción: 15 de Junio de 2015. Fecha de aprobación: 4 de Diciembre de 2015

2. Enfermera, Msc Directora del programa de Enfermería, Universidad Francisco de Paula Santander. Correo electrónico: dorisparada@ufps.edu.co

3. Estudiante de Enfermeria, Universidad Francisco de Paula Santander. Correo electrónico: leydi.2909@hotmail.com

4. Estudiante de Enfermeria, Universidad Francisco de Paula Santander. Correo electrónico: yfcardenas18@hotmail.com 


\section{Resumen}

Según el Ministerio de Educación Nacional de Colombia, el nivel de formación universitaria posee una tasa de graduación baja; sin embargo, los resultados varían en las diferentes regiones del país y las cifras de deserción son alarmantes. Objetivo: Determinar los factores relacionados con la permanencia estudiantil en los programas de pregrado presencial ofertados por la Universidad Francisco de Paula Santander, institución de carácter público situada en Cúcuta, ciudad fronteriza con Venezuela. Método: Enfoque cuantitativo descriptivo transversal con una muestra de doscientos dieciséis (216) estudiantes de último semestre. El instrumento es la encuesta denominada "Factores relacionados con la permanencia estudiantil en los programas académicos de pregrado presencial", diseñada y validada por las autoras. Resultados: Se halló que de los factores analizados (personales, socioeconómicos, institucionales y académicos), el de mayor influencia es el relacionado con lo personal. Conclusiones: Se evidencian aspectos influyentes en el logro de la permanencia, que deberían tener en cuenta las Instituciones Educativas, a fin de promover la permanencia y disminuir el rezago y la deserción de sus estudiantes; entre ellos se encuentran: la motivación y el gusto por la carrera seleccionada; la definición de un método de estudio; el aporte económico de los padres, aunque trabajar mientras se estudia cuando se tiene un propósito claro también es relevante; la calidad del programa académico; la metodología del docente; así como los recursos bibliográficos e informáticos a los que se tiene acceso.

Palabras clave: escolaridad; graduación; motivación; educación profesional

\section{Factors related to student retention in undergraduate programs in public universities}

\section{Abstract}

According to the Ministry of National Education of Colombia, Programs at the level of university education have a low graduation rate and dropout numbers are alarming. However, results vary in different regions of the country. Objective: To determine the factors related to student retention in face-to-face undergraduate programs offered by the Francisco de Paula Santander University, a public institution located in Cúcuta, a town bordering Venezuela. Method: Descriptive cross Quantitative approach with a sample of two hundred and sixteen (216) students from last semester. The instrument is a survey called "factors related to student retention in face-to-face undergraduate programs", designed and validated by the authors. Results: It was found that from the analyzed factors (personal, socio-economic, institutional and academic), the most influential is the one related to the personal. Conclusions: influential aspects are evident in achieving retention, which should be taken into account by the educational institutions in order to promote retention and reduce the backlog and desertion of their students; among them they are: motivation and how passionate the students are about the selected career; the definition of a method of study; the economic contribution of parents, although working while studying when you have a clear purpose is also relevant; the quality of the academic program; teaching methodology; as well as bibliographic and computer resources that are accessed.

Keywords: education; graduation; motivation; professional education 


\section{Fatores relacionados à retenção de alunos nos cursos de graduação de uma universidade pública}

\section{Resumo}

Segundo o Ministério de Educação Nacional da Colômbia, o nivel de formação universitária tem taxa de concluintes baixa; no entanto, os resultados flutuam nas diferentes regiões do país e os números de deserção são alarmantes. Objetivo: Determinar os fatores relacionados à retenção de alunos nos cursos de formação presencial ofertados pela Universidade Francisco de Paula Santander, instituição de caráter público localizada em Cúcuta, cidade fronteiriça com Venezuela. Método: Enfoque quantitativo descritivo transversal com amostra de duzentos e dezesseis (216) alunos de último semestre. $\mathrm{O}$ instrumento foi o inquérito nomeado "Fatores relacionados à retenção estudantil nos programas acadêmicos de formação presencial", desenhada e avaliada pelas autoras. Resultados: Descobriu-se que dos fatores analisados (pessoal, socioeconômico, institucional e acadêmico), o de maior influencia foi o relacionado com a questão pessoal. Conclusões: Foram evidenciados aspectos influentes para conseguir a retenção, a serem levados em conta pelas Instituições de Ensino, a fim de promover a permanência e diminuir o retraso e saída dos alunos; entre eles estão: motivação e gosto pela carreira escolhida; definição de um método de estudo; contribuição económica dos pais, ainda que trabalhar entanto estudar quando houver um propósito claro é mesmo relevante; qualidade do programa acadêmico; metodologia do docente; bem como os recursos bibliográficos e informáticos a que é possivel ter acesso.

Palavras-chave: nivel de instrução; formatura; motivação; ensino profissional 


\section{Introducción}

Como lo plantea la Ley 30 de 1992 que regula el Servicio Público de Educación Superior, la calidad del servicio educativo debe realizar inspección y vigilancia de las características generales más importantes en este proceso (1). En este sentido, la permanencia como uno de los elementos que integran el contexto de la calidad en la educación, debe ser evaluada y mejorada; pues como lo referencia Riascos (2), es la condición por la cual el estudiante se mantiene como miembro activo sin abandonar sus estudios en el tiempo programado sin desertar, lo cual además debe ser el objetivo de la institución educativa y promovido a través de estrategias para su logro.

La permanencia en educación superior, es un indicador de calidad que se relaciona con el cumplimiento de los logros de la politica de cobertura educativa, las metas de las políticas de calidad, pertinencia y eficiencia educativa del Sistema de Aseguramiento de la Calidad de la Educación Superior (3). No obstante, a pesar de las acciones desarrolladas para fomentar la permanencia y disminuir la deserción se han presentado dificultades en este logro a nivel mundial. Tinto, citado por Pineda en el documento: La voz del estudiante: el éxito de programas de retención universitario; refiere que países desarrollados como Estados Unidos reportan variación en sus tasas de graduación, en donde universidades como Harvard y Princeton consideradas como unas de las más prestigiosas del país norteamericano gradúan aproximadamente al 90\% de sus estudiantes, mientras que otras de carácter público, como las Universidad de Michigan o de California, gradúan el 80\%, y otras menos del 30\% de los estudiantes matriculados (4).

En América Latina, según la Organización de las Naciones Unidas para la Educación, la Ciencia y la Cultura (UNESCO) y el Instituto Internacional para la Educación Superior en América Latina y el Caribe (IESALC) (5), se gradúan en un periodo normal cerca del $43 \%$ de los estudiantes que ingresan, indicando que el $57 \%$ restante deserta o se demora mayor tiempo para graduarse.

En Colombia, de acuerdo con el Ministerio de Educación, la tasa de graduación en el nivel de formación universitario concentra un porcentaje del 33,6\% (6), de esto puede deducirse que las estrategias de retención estudiantil no han sido adoptadas o que ha faltado promoción de los programas que favorecen la permanencia en las Instituciones de Educación superior. Ha sido tan relevante esta problemática en el país, que ha llevado a plantear nuevas estrategias y como meta para el año 2019, se propone disminuir la actual tasa de deserción a 25\% (3). Uno de los departamentos con mayores índices de deserción es Norte de Santander (51.6\%), ocupando el segundo puesto después de la Guajira $(54,4 \%)$, con una tasa que supera el promedio nacional (45,3\%) (6); esto revela la necesidad de evaluar particularmente los diversos factores de tipo individual, social, académico e institucional que interfieren con la permanencia. Por otra parte, en cuanto a la tasa de deserción acumulada por cohorte a décimo semestre de las universidades de Norte de Santander, la Universidad Francisco de Paula Santander-Cúcuta llega al 52\%, representando cifras muy por encima de la media nacional (7). 
Al analizar la problemática, se evidencia la existencia de cuatro dimensiones o factores que se relacionan con la permanencia estudiantil; los aspectos personales o características individuales del estudiante; las variables socioeconómicas; las características institucionales; y por último, los factores académicos (3).

La clasificación anterior permite dilucidar este preocupante fenómeno y obtener información de manera organizada para el diseño de estrategias que fomenten la permanencia estudiantil en los estudiantes de pregrado, lo cual es centro de interés en el desarrollo de este estudio.

\section{Método}

Esta investigación es de tipo cuantitativo, descriptivo y de corte transversal. Para su desarrollo se identifican los factores relacionados con la permanencia estudiantil en los programas académicos de Pregrado Presencial que oferta la Universidad Francisco de Paula Santander en el segundo semestre de 2014.

La población fue conformada por estudiantes de último semestre, seleccionando aquellos que cumplieron los siguientes criterios de inclusión: estar matriculados durante el II semestre del 2014, que hubiesen permanecido en la institución sin realizar cancelación de semestre en algún periodo y que desarrollaran su programa académico hasta el último semestre en el tiempo programado, de acuerdo al plan de estudios de las 5 facultades de la Universidad. A partir de la información obtenida y del total de estos estudiantes, se extrajo una muestra por programas académicos, aplicando un nivel de confianza de $95 \%$, un error de $0,05 \%$ y una probabilidad de que ocurra el suceso de 50\%, obteniendo finalmente un total de 216 personas que integran el estudio.

El instrumento utilizado se denomina "Factores relacionados con la permanencia estudiantil en los programas académicos de pregrado presencial", diseñado por las autoras a partir de la revisión del estado del arte y validado a través de pruebas psicométricas. La validación de contenido se realizó por medio de la revisión multidisciplinar de expertos; lo anterior permitió la inclusión y eliminación de preguntas y validó la comprensión, duración y claridad de las mismas. Posterior a este proceso, se realizó una prueba piloto que determinó finalmente las preguntas requeridas y su forma de presentarlas. La prueba de fiabilidad obtuvo un coeficiente Alpha de Cronbach de 0,938 ; lo que demuestra que la definición operacional de las variables establecidas refleja realmente el significado teórico del problema estudiado.

El instrumento está integrado por dos áreas a diligenciar, una que contiene 20 preguntas correspondientes a información personal y 36 de tipo cerradas con respuestas en escala de Likert, referentes a los factores evaluados (9 ítems de factor personal, 4 ítems de factores socioeconómicos, 12 ítems de carácter institucional y 11 ítems de aspectos académicos). El rango de respuesta de cada aspecto se encuentra entre 1 y 5 , teniendo en cuenta el mayor o menor nivel de acuerdo con cada item presentado. Para evaluar el porcentaje de influencia dado a cada factor, los resultados se agruparon por rangos de acuerdo al número de ítems y valor asignado a cada uno de estos. 
Los participantes con previa información del estudio y de sus objetivos, otorgaron el consentimiento informado de manera escrita, adoptando para esta investigación los principios bioéticos establecidos por la Resolución $\mathrm{N}^{\circ} 008430$ de 1993. Para la tabulación y análisis, se realizó una base de datos en Excel y se aplicó el paquete estadístico SPSS versión 19, utilizando medidas estadísticas descriptivas y pruebas de relación como Chi cuadrado de Pearson para el análisis de algunas variables.

\section{Resultados}

\section{Características sociodemográficas}

En forma general, el rango de edad de la población de estudio está entre los 19 y los 32 años, siendo la edad de mayor frecuencia los 21 años (20,01\%), y presentándose en menor proporción los 32 años $(0,83 \%)$, con tendencia a disminuir la probabilidad de graduarse en los menores de 21 y mayores de 25 años.

Lo anterior demuestra que los estudiantes, en su mayoría, terminan su carrera a una edad joven. Estos hallazgos son similares y confluyen con lo referido por Vergel (7), quien refiere que las edades inferiores a 16 años y superiores a 30 años disminuyen la probabilidad de permanencia, aspecto que se observa en menor proporción en el presente estudio.

El género de los estudiantes es masculino en su mayoría $(54,24 \%)$, dato que difiere s discordantes con los resultados de Barrios (8) y de Leone y Tian (9), que hallaron mayor proporción del género femenino en sus resultados. Otros estudios como el de Laskey y Hetzel (10) y el de Fontes de Souza y Núñez da Silva (11), indican que no hay diferencia significativa en la permanencia por motivos de género.

Con respecto al estado civil de la población de estudio, en su mayoría son solteros $(85,2 \%)$ y en un segundo lugar aparece la unión libre como la condición prevalente $(8,1 \%)$. Similar a los resultados de Barrios (8) y Gutiérrez (12), en los cuales se detalla que los solteros ocupaban el mayor porcentaje de la población estudiada. En este contexto, los resultados destacan la relevancia que tiene la soltería para alcanzar las metas académicas propuestas.

La población objeto de estudio, corresponde al estrato socioeconómico $2(46,13 \%)$ y $3(34,69 \%)$. Lo anterior se relaciona con la naturaleza jurídica de la universidad en la cual realizan sus estudios, la cual es pública y acoge a la mayoría de estudiantes de bajos recursos económicos.

Con respecto a la vivienda y el tipo de familia, se encuentra que el $(70,8 \%)$ refiere vivir con los padres, un $(11,8 \%)$ con otros familiares o familia extensa y; en menor proporción se halló a quienes comparten sus viviendas con una familia reconstruida en la que se integran padrastros o madrastras $(0,4 \%)$. Resultados coherentes con los hallazgos de Mares está al., en México (13), y Gutiérrez en Colombia (12), en donde la mayoría de la población de 
estudio refirió vivir con sus padres y hermanos. Estos hallazgos demuestran que el convivir en una familia nuclear puede ser un factor protector que permite el alcance de metas académicas.

Al indagar la formación académica de los padres, en su mayoría se halló que $45 \%$ posee bachillerato y el $22.69 \%$ primaria; solo el $18 \%$ de los padres tienen un nivel académico de pregrado. Los hallazgos del presente estudio permiten analizar que el nivel educativo de los padres puede ser un factor que coadyuva a que sus hijos permanezcan en los estudios, siendo el bachillerato un grado considerable de instrucción que posee el padre o madre del estudiante.

Con respecto a la situación laboral durante sus estudios, se identificó que el 34\% trabajaba; dentro de las actividades realizadas el $18,82 \%$ lo hacía como independiente, el (15,50\%) fue empleado en trabajos con horarios contrarios a sus jornadas académicas, y en menor proporción un porcentaje se dedicaba a las labores del hogar. En relación con esta información, Giovagnoli PI, citado por Herrero, Merlino, Ayllon y Escanes (14) indica que para un estudiante que está trabajando, al menos durante el último año de universidad, la probabilidad de graduarse es $45 \%$ menor que para uno con tiempo completo dedicado al estudio, como puede identificarse en los resultados del presente estudio, puesto que de los estudiantes próximos a graduarse solo existe un pequeño porcentaje que logró llegar a esta meta mientras trabajaba.

Pudo identificarse en relación a la variable: tener personas a su cargo, que el $13,7 \%$ tuvo una persona durante su etapa de estudio universitario, mientras que el 0,8\% asumió 4 o más. De estos hallazgos se puede inferir que para estas personas esta responsabilidad adicional implica un grado más de sobrecarga y esfuerzo. No obstante, Fontes de Souza, Nunes da Silva (11), Castaño, Gallon y Gomez, citados por Pineda y Pedraza (15), identifican esta variable como una de las razones que mejoran la permanencia por el sentido de responsabilidad que se debe poseer si se desea culminar con éxito.

\section{Factores relacionados con la Permanencia Estudiantil}

\section{Factor personal o individual}

Al indagar sobre este factor, se encontró que para el 88,56\% de los encuestados, los aspectos que lo integran tienen alta influencia en su decisión de permanecer en la universidad hasta culminar la carrera, y que variables como la motivación personal, la vocación y el gusto por la carrera escogida, así como tener una familia unida y llevar a cabo una técnica o método de estudio, representa para el estudiante, variables de importancia para continuar su formación académica con éxito dentro de la institución, como puede verse en el siguiente gráfico. 


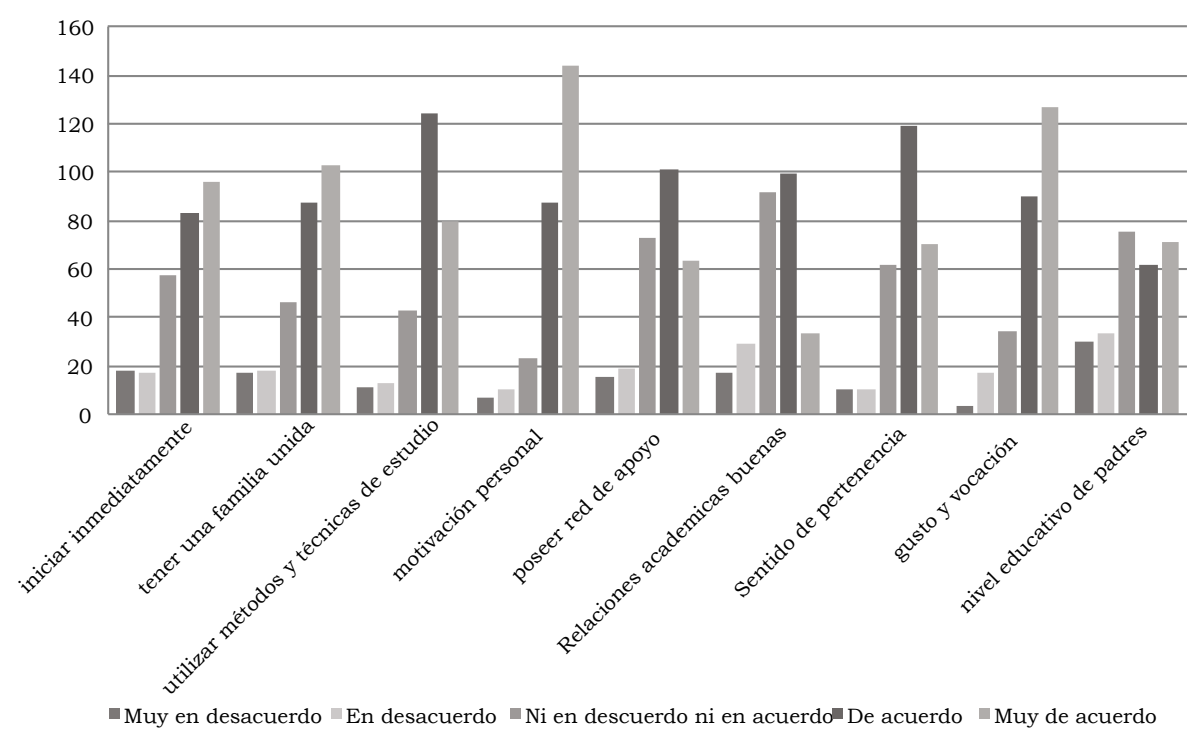

Grafico $\mathbf{N}^{\circ} \mathbf{1}$. Aspectos del factor personal relacionados con la permanencia en los programas de pregrado presencial, UFPS, 2014

Fuente: Instrumento Factores relacionados con la permanencia estudiantil en los programas académicos de pregrado presencial, 2014. Elaboración propia.

Estos resultados, son similares a los hallazgos de Fernández et ál, (16) en donde se concluye que los estudiantes universitarios que utilizan estrategias de aprendizaje más complejas, presentan significativamente mejor rendimiento académico, y significativamente menos reprobaciones que el grupo de estudiantes que utilizan estrategias de aprendizaje más simples.

Diferentes estudios han identificado que el método de estudio, uso inadecuado del tiempo y falta de motivación representan causas que influyen en la decisión de retirarse de la institución (17) y que factores asociados al bajo rendimiento académico son el estado emocional seguido de la situación familiar disfuncional (13).

Por otra parte, el iniciar la carrera inmediatamente después de terminar el bachillerato no representó influencia alguna con la probabilidad de que el estudiante permaneciera hasta culminar sus estudios, similar a la investigación realizada por Giovagnoli, (14), en la que se determinó que egresar de la secundaria e iniciar al año siguiente la carrera de grado no parece contribuir significativamente a una mayor probabilidad condicional de culminar exitosamente los estudios universitarios.

Los hallazgos de este factor reflejan la importancia de fomentar en el estudiante el aprendizaje de métodos y técnicas de estudio, así como talleres de motivación en los primeros semestres académicos, siendo estos la base del afianzamiento con el contexto universitario, con el fin contribuir a optimar su desempeño y adherencia para continuar los estudios, lo cual permite al estudiante su visualización como profesional en el campo 
laboral, fortalece su rendimiento académico y por ende su permanencia dentro de la institución.

\section{Factor socioeconómico}

En el factor socioeconómico, se destacan de manera individual aspectos que fueron relevantes para lograr la permanencia en su carrera de pregrado, como el aporte recibido de su familia $(76 \%)$ o estudiar y trabajar simultáneamente (49\%). Resultados que pueden observarse en la siguiente gráfica.

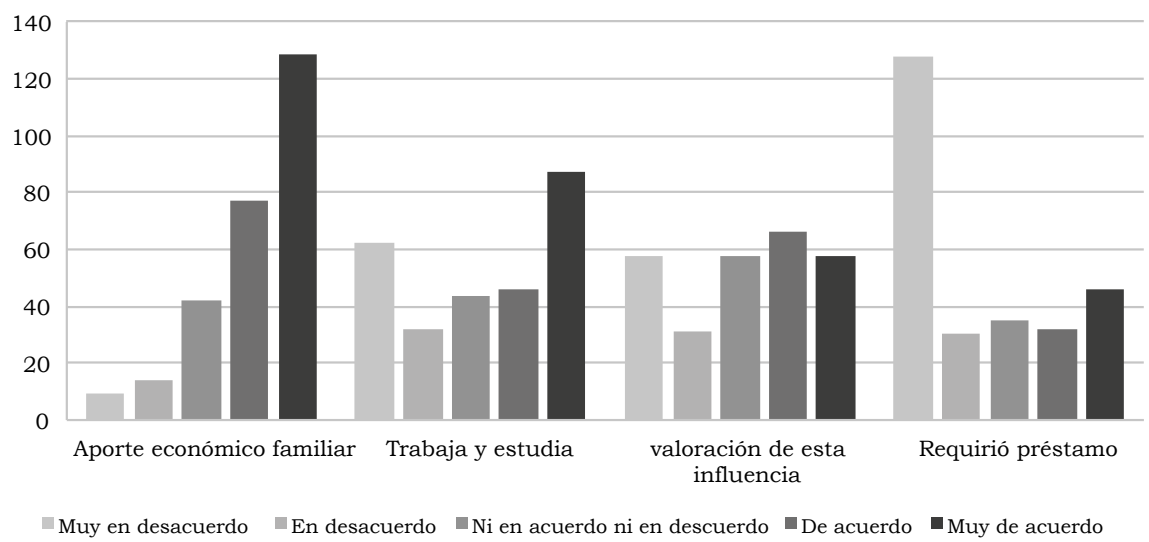

Grafica $\mathbf{N}^{\circ}$ 2. Aspectos socioeconómicos relacionados con la permanencia en el pregrado, UFPS, 2014

Fuente: Instrumento Factores relacionados con la permanencia estudiantil en los programas académicos de pregrado presencial, 2014. Elaboración propia.

En forma general este factor fue identificado como preponderante por un $(53,5 \%)$ de la población encuestada, lo que permite deducir que para este grupo, las variables presentadas ejercen una relación importante para permanecer en la institución. Algunos refieren que trabajar mientras se estudia "es pesado" e interfiere con el cumplimiento de sus obligaciones académicas, pero que los hace sentir mayor responsabilidad para cumplir la meta de graduarse. Al realizar el análisis entre el género y la variable influencia positiva del trabajo sobre la permanencia estudiantil, se obtuvo un $\mathrm{X}^{2}$ con un valor de $(4)=9.64, \mathrm{p}<0.05$, evidenciándose una relación estadísticamente significativa entre estos, siendo para los hombres un aspecto que fomenta su logro.

Similar a los resultados del estudio de Gutiérrez (12), en el cual se concluye que el factor socioeconómico representa mayor influencia en la 
permanencia estudiantil. Difiere por el contrario a los resultados del estudio de Martínez, en el cual refiere que la situación económica no influye en la decisión de desertar de la universidad. Los hallazgos actuales permiten evaluar que esta variable puede estar unida a otras como el contexto familiar y requerimientos de la academia, por lo que se generan dos grupos casi homogéneos que refieren diversas opiniones.

\section{Factor institucional}

Los aspectos evaluados individualmente dentro de este factor y su valoración positiva como influyente en la permanencia estudiantil fueron la calidad del proceso de formación (62\%), la modalidad presencial (67\%), las prácticas pedagógicas utilizadas por los docentes (53\%), así como las estrategias de evaluación (53\%), como puede verse en los resultados presentados en la gráfica $\mathrm{N}^{\circ} 3$.

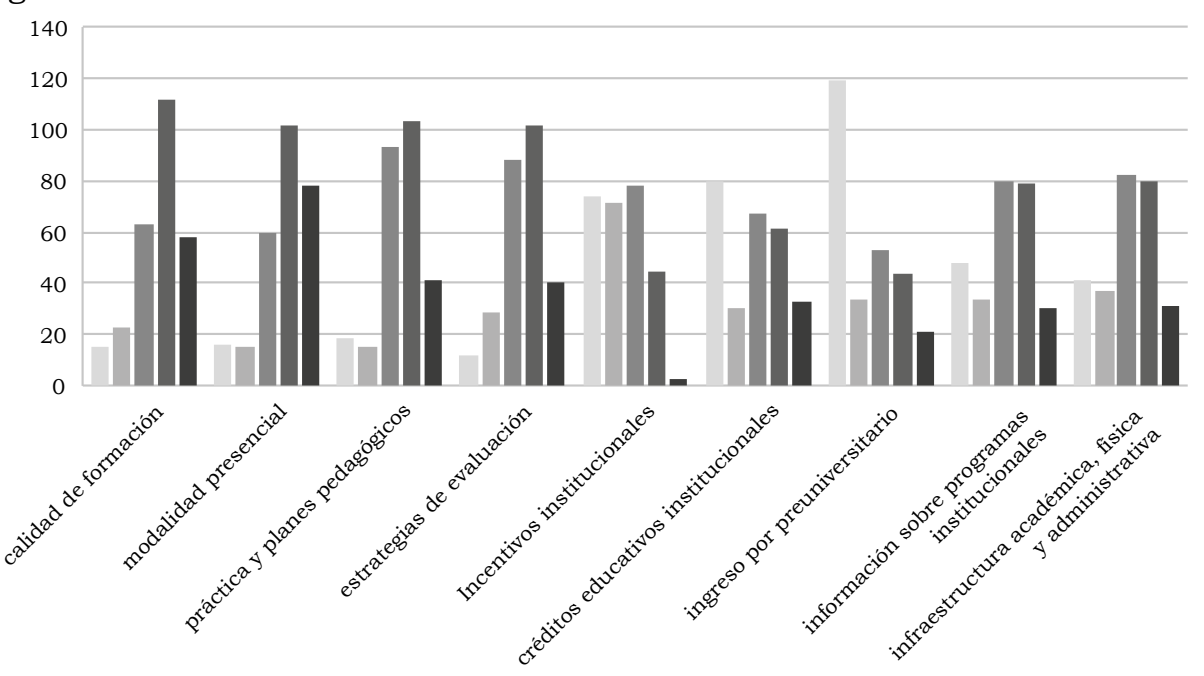

Muy en desacuerdo $\square$ En desacuerdo $\square$ Ni en acuerdo ni en desacuerdo $\square$ De acuerdo $\square$ Muy de acuerdo

Gráfica $\mathbf{N}^{\circ}$ 3. Aspectos institucionales que influyen con la permanencia en el pregrado, UFPS, 2014.

Fuente: Instrumento Factores relacionados con la permanencia estudiantil en los programas académicos de pregrado presencial, 2014. Elaboración propia.

Es importante observar que para el estudiante que logra su objetivo académico en el tiempo programado se hace relevante la práctica pedagógica utilizada por el docente en el proceso de enseñanza aprendizaje, entendiendo 
que dentro de este se da el encuentro de saberes entre docentes y estudiantes a través de la interacción y de la comunicación pedagógica, como lo referencia Granja (18), lo cual hace necesario fijar la mirada sobre este aspecto e identificar ¿Cuál es el proceso que se está dando al respecto en cada programa académico? Analizar a su vez el lugar donde dichos saberes tienen lugar en la práctica pedagógica: ¿Cómo se comunica al estudiante esta información y cómo se construye el conocimiento para que realmente sea importante, heurístico y aplicable al contexto actual?

Para el 50,9\% de la población de estudio, este factor presenta una influencia positiva en el logro de la meta profesional y la permanencia en la universidad. Este hallazgo permite identificar que existe una división casi proporcional en los resultados positivos y negativos con respecto a este factor y que puede estar relacionado con las diferencias de calidad en los diversos programas.

Al respecto Barbosa $\mathrm{T}$ halló en su estudio realizado en el programa Enfermería de la misma Universidad, el cual tiene acreditación de alta calidad, que la causa en menor proporción que impide la permanencia en el programa, es de índole institucional. Otros hallazgos como el de Umfres (19) han demostrado que la variable institucional ha sido el predictor más fuerte de retención de los estudiantes.

Los hallazgos actuales reflejan la importancia de fortalecer estos aspectos institucionales que conllevan a aumentar el porcentaje de permanencia en los programas de educación superior entre los cuales se puede identificar la necesidad de adoptar o mejorar un programa de inducción, así como de difundir los incentivos y beneficios institucionales al estudiante al iniciar su pregrado, puesto que se ha evidenciado además por otros autores como Corral y Díaz (20), que el motivo más influyente en el factor institucional para desertar es el desconocimiento del plan de estudio.

\section{Factor académico}

Dentro de los aspectos que integran este factor, se identificaron variables positivas relacionadas con la permanencia estudiantil como el contar con recursos informáticos y bibliográficos en el programa (61\%) y la relación de la carga académica (51\%); es decir, la oportunidad que tiene el estudiante de acceder a las salas de biblioteca y de informática para adquirir estos recursos y la relevancia de disponer del tiempo para su estudio y descanso en relación con la permanencia en la Institución educativa. Véase resultados en la gráfica $\mathrm{N}^{\circ} 4$.

Los resultados demuestran la necesidad de incrementar los recursos bibliográficos, informáticos y laboratorios en los programas académicos, así como de mejorar su uso y el acceso a los mismos, los cuales son criterios establecidos por el Consejo Nacional de acreditación para medir la calidad que oferta el programa a sus estudiantes. 


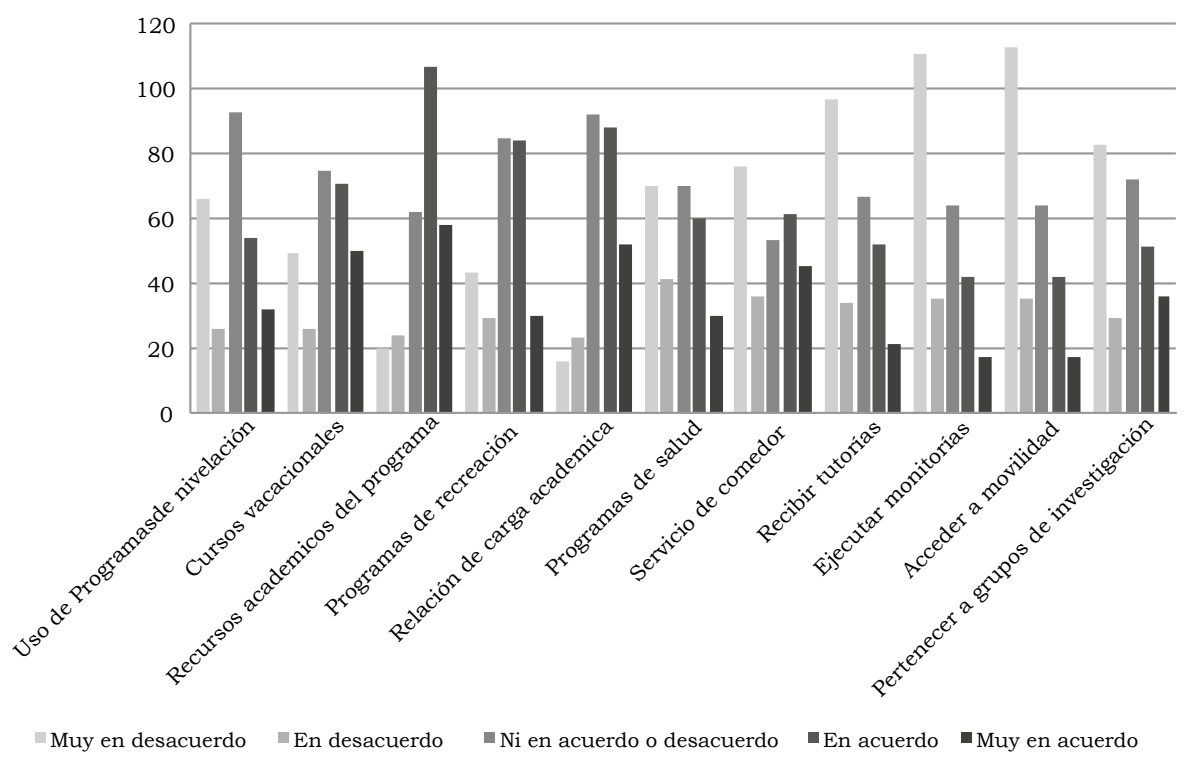

Gráfica $\mathbf{N}^{\circ}$ 4. Aspectos académicos relacionados con la permanencia en el pregrado, UFPS, 2014

Fuente: Instrumento Factores relacionados con la permanencia estudiantil en los programas académicos de pregrado presencial, 2014. Elaboración propia.

Este hallazgo es similar a los resultados de Mares (13), quien encontró que los recursos insuficientes en la biblioteca, la ausencia de equipos audiovisuales, las técnicas didácticas y la poca capacidad en el uso de recursos educativos afecta la motivación del estudiante, y por tanto, su permanencia en la educación superior.

Debe resaltarse que no todos los programas de la institución cuentan con tutorias para el estudiante, por lo cual esta estrategia es reconocida solamente por aquellos que cuentan con este apoyo educativo. Este aspecto es un factor relevante para alcanzar permanencia, ampliamente demostrado en diversos estudios. Por ejemplo, en los estudios de Laskey y Hetzel (10), y Ramos, Machado y Monteiro (21), se indica que para evitar la deserción de los estudiantes, las tutorias con pares son un mecanismo muy importante.

De igual forma, el Ministerio de Educación Nacional y la Universidad de Antioquia (22) en el trabajo denominado Proyecto Bienestar, Equidad y Permanencia Estudiantil, identificó que algunos estudiantes requieren de un proceso de identificación para ser beneficiarios de procesos de tutoría y acompañamiento académico extracurricular.

Respecto a los servicios del bienestar universitario, en concordancia con los hallazgos de Gutiérrez (12), se encontró que no están calificados por los estudiantes como relevantes en relación con la permanencia estudiantil, situación causada probablemente a la ausencia de información/promoción, falta de tiempo y/o motivación para asistir a estos servicios. 


\section{Conclusiones}

En el análisis de la relación de factores y la permanencia estudiantil, se evidencia que el factor personal integra los aspectos de mayor relevancia para este logro académico, haciendo notables los siguientes aspectos: la motivación personal por estudiar, el gusto o la vocación por la carrera escogida, el tener a su familia unida y el uso de una técnica o método de estudio adecuado para el desarrollo de su formación intelectual. Lo cual permite identificar estos aspectos como factores protectores para permanecer hasta graduarse en el tiempo ideal.

No obstante existen aspectos de tipo institucional y académico que evidencian una relación con la permanencia como logro académico.

En ese sentido se deben resaltar los siguientes aspectos: La calidad del plan de estudio evidenciando la importancia de poseer acreditación de alta calidad; la modalidad presencial; el método pedagógico que lleva a cabo el docente en las aulas de clase y fuera de éstas, que converge en la interacción y comunicación exitosa con el estudiante; y por último, el contar con acceso constante a los recursos académicos brindados por la Universidad, particularmente el servicio de la biblioteca y las salas de cómputo.

\section{Financiamiento}

Ninguno

\section{Conflicto de intereses}

No existe

\section{Referencias}

1. Colombia. Ministerio de Educación Nacional. Ley 30 de diciembre de 1992. [Internet] [Citado 14 Marzo 2014]. Disponible en: http://www.mineducacion.gov.co/1621/article-86437.html.

2. Riascos VH. Metodología para la evaluación de las políticas, estrategias y proyectos de permanencia estudiantil en UNINCCA. Bogotá. [Internet]. 2012. [Citado 15 Abril 2014] Disponible en: https://www.unincca.edu. $\mathrm{co} /$ permanencia/plandedesarrollo/documentos/cartillametodologia.pdf

3. Colombia. Ministerio de Educación Nacional. Deserción Estudiantil en la Educación Superior Colombiana: Metodología de Seguimiento, diagnóstico y elementos para su prevención. [Internet]. 2009 [Citado 10 Febrero 2014]. Disponible en: http://www.mineducacion.gov.co/sistemasdeinformacion/1735/articles-254702_libro_desercion.pdf.

4. Pineda C. La voz del estudiante: el éxito de programas de retención universitaria. Colombia: Universidad de La Sabana, UNESCO, IESALC: 16 [Internet]. 2010 [Citado 1 marzo 2014] Disponible en: https://books. google.com.co/books?id=RsAlx557jx0C\&pg=PA7\&lpg=PA7\&dq=La+voz + del+estudiante:+el+\%C3\%A9xito+de+programas + de + retenci $\% \mathrm{C} 3 \% \mathrm{~B} 3$ $\mathrm{n}+$ universitaria.\&source $=\mathrm{bl} \& \mathrm{ots}=\mathrm{m}-8 \mathrm{FWAMjqY \& sig}=\mathrm{EOD} 9 \mathrm{cukvP} 4 \mathrm{~kb}-$ 7QoRNQ9VBfG5xc\&hl=es\&sa=X\&ved=0CCsQ6AEwAmoVChMI 7t-10Mz1xwIVhyYeCh3DVQ5f\#v=onepage\&q\&f=false 
5. Instituto Internacional para la Educación Superior en América Latina y el Caribe IESALC. Rezago y Deserción en la Educación Superior: Centro Interuniversitario de Desarrollo-CINDA- IESALC: Universidad de Talca Chile. [Internet].- 2006 [Citado 5 marzo 2014] Disponible en: http:// www.universidadfutura.org/wp-content/uploads/2012/05/Repitenciay-Deserci\%C3\%B3n-Universitaria-en-Am\%C3\%A9rica-Latina1.pdf

6. Colombia. Ministerio de Educación Nacional. Diagnóstico de Deserción. Departamentos de Santander y Norte de Santander.[Internet].2012 [Citado 12 marzo 2014] Disponible en: http://www.mineducacion.gov.co/1621/ articles-302596_archivo_pdf_diagnostico_desercion_santander.pdf

7. Vergel M. Hacia un modelo para evaluar la pertinencia social en la oferta académica de la Universidad Francisco de Paula Santander. [Tesis de Doctorado] San José de Cúcuta: Universidad Francisco de Paula Santander: 2013.

8. Barrios A. Deserción Universitaria en Chile: Incidencia del financiamiento y otros factores asociados. [Internet] 2011. [Consultado el día 20 de enero de 2014] Disponible en: http://www.techo.org/wp-content/ uploads/2013/02/barrios.pdf

9. Leone M, Tian R. Push vs pull: Factors influence student retention. Rev American Journal of Economics and Business Administration. 2009, Volumen 1(2): 122-132. [Internet] [Citado el 8 nov 2014] Disponible en: http://search.proquest.com/docview/1027115302?accountid=43636

10. Laskey M, Hetzel C. Investigating Factors Related to Retention of AtRisk College Students. Journal Learning Assistance Review. Volumen 16, 159 (1): 31-43. [Internet]. 2011 [Consultado 06 nov 2014] Disponible en: http://files.eric.ed.gov/fulltext/EJ919577.pdf

11. Fontes de Souza, AL, Nunes da Silva, M. Uma Investigação Sobre Os Fatores Contribuientes Na Retenção Dos Alunos No Curso De Ciências Contábeis Em Uma Ifes: Umdesafio À Gestão Universitária. ReCont: Registro Contábil. Vol 2 (3). Brasil. [Internet]. 2011. [Consultado 7 nov 2014] Disponible en: http://www.seer.ufal.br/index.php/registrocontabil/ article/view/511/266

12. Gutiérrez Á. Programa de Permanencia Estudiantil para el plan de estudios de Administración de Empresas de la Universidad Francisco de Paula Santander, Cúcuta. [Internet]. 2013 [Consultado el día 15 de octubre de 2014] Disponible en: https://prezi.com/dwfqkjb40g9t/ permanencia-fce-ufps/

13. Mares $\mathrm{G}$, et ál. Identificación de factores vinculados con la deserción y la trayectoria académica de los estudiantes de psicología en la FES Iztacala. Red Mexicana de Investigación en psicología. [Internet] [[Citado el dia 13 de septiembre de 2015] Disponible en: http://www.cneip.org/ documentos/12.pdf

14. Giovanoli, [citado por Herrero V, Merlino A, Ayllon S, Escanes G]. Aplicación de un modelo de duración en programas de prevención de deserción universitaria. Rev Electrónica de Investigación Educativa. Vol 15 (3) [Internet]. 2013 [Consultado el día 15 de enero de 2014] Disponible en: http://webcache.googleusercontent.com/search?q=cache:PM1wZsC 
P9MIJ:redie.uabc.mx/index.php/redie/article/download/554/838+\&cd $=8 \& \mathrm{hl}=\mathrm{es} \& \mathrm{ct}=\mathrm{clnk} \& \mathrm{gl}=\mathrm{co}$

15. Castaño E, Gallón S, Gómez K, [citados por Pineda y Pedraza]. Persistencia y graduación: Hacia un modelo de retención estudiantil para instituciones de Educación Superior. [Internet]. 2011 [Citado el 1 febrero 2015]. Disponible en: https://books.google.com.co/books?id=-VUg5ypT9VkC\&p $\mathrm{g}=\mathrm{PA} 169 \% 1 \mathrm{pg}=\mathrm{PA} 169 \% \mathrm{dq}=\mathrm{An} \% \mathrm{C} 3 \%$ A1lisis + De + Los + Factores + Asociados $+\mathrm{a}$ +la+Deserci\%C3\%B3n+y+Graduaci\%C3\%B3n+Estudiantil+Universitaria. +Lecturas+de+Econom\%C3\%ADa\&source=bl\&ots=kHQnYcyufV\&sig=_v9 aZSyxEWzsXlqz5070dU3EQrk\&hl=es\&sa=X\&ved=0CFQQ6AEwB2oVCh MIlq_XtNr1xwIVjbIeCh11nwRr\#v=onepage $\& q=A n \% C 3 \% A 11$ isis $\% 20 D e \% 20$ Los\%20Factores\%20Asociados\%20a\%20la\%20Deserci\%C3\%B3n\%20 y\%20Graduaci\%C3\%B3n\%20Estudiantil\%20Universitaria.\%20 Lecturas\%20de\%20Econom\%C3\%ADa\&f=false

16. Fernández O, Martínez M, Melipillan A. Estrategias de Aprendizaje y Autoestima. Su Relación con la Permanencia y Deserción Universitaria. Rev Estudios Pedagógicos (Valdivia). Volumen 35 (1) [Internet. 2009 [Consultado 1 febrero del 2014]. Disponible en: http://www.redalyc. org/articulo.oa?id=173514138002

17. Barbosa Trillos, Lizbelky Viviana. Factores Relacionados con la Deserción y Rezago de los estudiantes del Programa de Enfermería de la Universidad Francisco De Paula Santander I 2009 - II 2011. [Tesis de pregrado] San José de Cúcuta: Universidad Francisco de Paula Santander: 2011.

18. Granja C. Caracterización de la comunicación pedagógica en la interacción docente-alumno. Rev Investigación en Enfermería: Imagen y desarrollo: Pontificia Universidad Javeriana. Vol 15 (2). [Internet]. 2013 [Consultado Julio del 2015]. Disponible en: http://www.redalyc.org/ pdf/1452/145229803005.pdf

19. Umfres J. Un análisis de gastos de asuntos estudiantiles: servicios y estudiante universitario de retención en cuatro años colegios y universidades en los Estados Unidos. ProQuest Internet 2010 [Consultado 06 de nov 2014] Disponible en: http://search.proquest.com/docview/304676077/ previewPDF?accountid $=43636$

20. Corral V, Díaz X. Factores asociados a la reprobación de los estudiantes de la universidad de Sonora. [Internet]. 2009 [Citado el día 01 de febrero de 2014] Disponible en: http://www.comie.org.mx/congreso/ memoriaelectronica/v10/pdf/area_tematica_16/ponencias/0910-F.pdf

21. Ramos M, Machado A, Monteiro M. Ações para o combate a retenção e evasão acadêmica e qualificação do ensino da graduação em Nutrição da Universidade Federal do RS. Universidade Federal Do Rio Grande Do Sul. $4^{\circ}$ andar - Porto Alegre. Brasil. [INTERNET]. 2010 [Consultado 7 nov 2014] Disponible en: http://www.lume.ufrgs.br/bitstream/handle/10183/62608/Ensino2011_Resumo_19084.pdf?sequence=1

22. Ministerio de educación Nacional, Universidad de Antioquía. Bienestar, equidad y permanencia estudiantil: seguimiento y evaluación. [INTERNET]. 2009 [Consultado 7 nov 2014] Disponible en: http://www.colombiaaprende.edu.co/html/micrositios/1752/articles-323093_recurso_1.pdf 
Doris Amparo Parada Rico et al.

23. Ministerio de Educación Nacional, M. d. Revolución Educativa Colombia Aprende. Deserción Estudiantil en la Educación Superior Colombiana, Metodología de Seguimiento, diagnóstico y elementos para su prevención. Colombia. pág. 149. [INTERNET] 2009 [Consultado 7 nov 2014] disponible en: http://www.mineducacion.gov.co/sistemasdeinforma 